\title{
A 25-Year-Old Man with Exudative Retinal Detachments and Infiltrates without Hematological or Neurological Findings Found to Have Relapsed Precursor T-Cell Acute Lymphoblastic Leukemia
}

\author{
Jordan S. Johnson $^{\mathrm{a}}$ James S. Lopez ${ }^{\mathrm{a}}$ Arthur Scott Kavanaugh ${ }^{\mathrm{a}}$ \\ Chanping Liang ${ }^{a}$ Douglas A. Mata ${ }^{b}$ \\ ${ }^{a}$ Department of Ophthalmology, Louisiana State University Health Sciences Center, \\ Shreveport, La., and 'Department of Pathology, Brigham and Women's Hospital, Harvard \\ Medical School, Boston, Mass., USA
}

\section{Key Words}

Leukemia · Retinal detachment - Ophthalmology

\begin{abstract}
Background: Precursor T-cell acute lymphoblastic leukemia (pre-T-ALL) may cause ocular pathologies such as cotton-wool spots, retinal hemorrhage, and less commonly, retinal detachment or leukemic infiltration of the retina itself. However, these findings are typically accompanied by the pathognomonic hematological signs of acute leukemia. Case Presentation: In this case report and review of the literature, we describe a particularly unusual case of a 25-year-old man who presented to our hospital with bilateral exudative retinal detachments associated with posterior pole thickening without any hematological or neurological findings. The patient, who had a history of previously treated pre-T-ALL in complete remission, was found to have leukemia cell infiltration on retinal biopsy. Conclusion: Our case underscores the fact that the ophthalmologist may be the first provider to detect the relapse of previously treated leukemia, and that ophthalmic evaluation is critical for detecting malignant ocular infiltrates.


Johnson et al.: Retinal Infiltrate with Exudative Retinal Detachment due to T-ALL Relapse

\section{Introduction}

Precursor T-cell acute lymphoblastic leukemia (pre-T-ALL) is an immune-cell neoplasm that commonly presents with ocular manifestations [1]. Retinopathies such as central retinal vein occlusion, intraretinal, white-centered, or vitreous hemorrhage, or leukemic infiltration are found in $12-29 \%$ of patients at the time of leukemia diagnosis [2, 3]. Unilateral exudative retinal detachment with associated leukemic infiltration, a remarkably rare ocular complication of pre-T-ALL, occurs infrequently. Such ocular pathologies typically arise in the setting of active leukemic disease and do not generally occur in patients in hematological remission [4]. Below, we report an unusual case of a man who presented with bilateral exudative retinal detachments with associated retinal leukemic infiltration as the sole sign of pre-T-ALL relapse after 4 months in remission.

\section{Case Presentation}

A 25-year-old man with high-risk pre-T-ALL in complete hematological remission for 4 months and no ophthalmic history presented to our hospital with 2 weeks of bilateral ocular pain with decreased vision and 1 week of right-sided ocular redness with periocular and temporoparietal headache. He reported no dizziness, dysphagia, fever, focal weakness, hearing loss, or paresthesia and had no history of radiation to the head. Inquiry about family history revealed blindness in his maternal grandmother. The patient had completed first-line hyper-CVAD chemotherapy, courses $1 \mathrm{~A}$ (i.e., cyclophosphamide, vincristine, doxorubicin, and dexamethasone) to 4B (i.e., methotrexate and cytarabine) in August 2014. He had also completed a 10-course regimen of prophylactic intrathecal chemotherapy in November 2014, which is the standard of care for pre-T-ALL. A post-treatment bone marrow biopsy was negative for residual disease.

On physical examination, the patient had hand-motion vision in both eyes. Tonometric right- and left-eye pressures were 38 and $16 \mathrm{~mm} \mathrm{Hg}$, respectively. Pupils were sluggishly reactive without afferent pupillary defects. Upward gaze limitation with convergence/retraction-type nystagmus was present. Other gaze fields were normal. The eyelids, lashes, and lacrimal glands were normal on slit-lamp examination. The right-eye conjunctiva was diffusely injected, while that of the left eye was white and quiet. Corneas were clear. The right-eye anterior chamber had a grade 2 flare, while that of the left eye was deep and quiet. Irises were normal and lenses were clear. On dilated fundus examination, the right- and lefteye vitreous were hazy and clear, respectively. Bilateral diffuse intraretinal hemorrhages with whitening and complete bilateral retinal detachments without identifiable tears were seen (fig. 1). Retinal folds and areas of bullous detachment were noted. Nerves were difficult to visualize. B-scan ultrasonography confirmed these findings and revealed significant retinal thickening at the posterior poles (fig. 2).

The patient was admitted and started on intravenous acyclovir to cover for possible acute retinal necrosis versus progressive outer retinal necrosis. As the initial complete blood count was normal, there was less concern for cytomegalovirus retinitis. The patient was started on dorzolamide/timolol for his increased right intraocular pressure. Given his upward gaze palsy, he underwent magnetic resonance imaging to assess for possible central nervous system involvement, which showed only soft-tissue masses consistent with subretinal exudative fluid (fig. 3). Lumbar puncture was normal. Right-eye vitreal biopsy polymerase chain reaction was negative for herpes simplex, varicella zoster, cytomegalovirus, and toxoplasmosis, and cytology was negative for malignancy. 
Johnson et al.: Retinal Infiltrate with Exudative Retinal Detachment due to T-ALL Relapse

Because the patient was not improving on antivirals, retinal biopsy was performed. As on dilated fundus examination, no tears were identified, and a diagnosis of bilateral exudative retinal detachment was confirmed. The patient underwent left-eye retinal detachment repair with biopsy. Pathologic analysis of the retinal biopsy, including molecular, cytogenetic, and flow cytometric testing, revealed infiltration with pre-T-ALL (fig. 4). This finding was confirmed on left-eye vitreal and subretinal fluid cytology. The patient was referred to his hematologist for further treatment with radiation therapy to both eyes, systemic chemotherapy, and bone-marrow transplant. The patient's exudative detachment and optic nerve symptoms improved. The right-eye intraocular pressure corrected, and the left-eye retina remained flat. Nonetheless, his visual acuity remained poor in both eyes, with light perception vision.

\section{Discussion}

Ocular manifestations occur in $12-29 \%$ of patients with pre-T-ALL and are more common in patients with high-risk leukemia (i.e., initial white blood cell counts over $500 \times 10^{9} / \mathrm{l}$ ) [2-4]. Orbital and ocular lesions secondary to leukemic infiltrates are associated with a higher frequency of bone marrow relapse and central nervous system involvement. Ocular findings typically occur secondary to direct leukemic infiltration of the ocular structures; hematological abnormalities such as anemia, hyperviscosity, leukopenia, or thrombocytopenia; opportunistic infections, or as complications of chemotherapy [5]. Cotton-wool spots and intraretinal hemorrhages are commonly seen manifestations, while exudative retinal detachments with leukemic infiltrates are unusual [6]. Intraocular leukemia is generally treated with intrathecal and systemic chemotherapy. If leukemic infiltrates do not respond to chemotherapy, ocular radiation may be utilized.

The mechanism of pre-T-ALL-induced exudative retinal detachment is thought to be caused by leukemia cell infiltration with subsequent occlusion of the choroidal capillaries and choroidal thickening. Ischemia-induced dysfunction of Bruch's membrane and the retinal pigment epithelium leads to retinal detachment. Aside from pre-T-ALL, other causes of retinal detachment include central serous chorioretinopathy, choroidal melanoma, choroidal neovascularization, congenital optic disc pits, Harada's syndrome, uveal effusion syndrome, severe ocular hypotony, cardiac disease, and renal dysfunction [7, 8]. In our case, these causes were excluded by the patient's history and ocular presentation.

Although exudative retinal detachment with leukemic infiltration as the sole manifestation of leukemia relapse is rare, cases have been reported in the literature. Primack et al. [9] described the case of a 3-year-old boy with ALL in remission who presented with retinal detachment and painful neovascular glaucoma. Enucleation was performed and pathologic analysis revealed leukemic infiltrates of the retina and optic nerve. To the best of our knowledge, this was the first reported case of leukemia relapse presenting with retinal detachment without other systemic signs. Schmiegelow et al. [10] described another case of ALL relapse with retinal detachment after 1 year of remission. Initial subretinal fluid biopsy via sclerotomy did not reveal malignant cells, and the patient was therefore treated with steroids and atropine. Five months later, the exudative detachment reoccurred and pars plana vitrectomy was performed. Subretinal biopsy showed leukemic infiltrate in the choroid.

Azik et al. [8] described a third case, an 8-year-old boy with a history of pre-B-ALL who presented with unilateral vision loss and exudative retinal detachment of the left eye with a subretinal mass. The patient did not undergo biopsy to confirm the diagnosis but rather re- 
Johnson et al.: Retinal Infiltrate with Exudative Retinal Detachment due to T-ALL Relapse

ceived local radiotherapy and systemic and intrathecal chemotherapy with resolution of the mass. Yoshida et al. [4] described a separate case of bullous exudative retinal detachment due to leukemic infiltrate in a child with T-ALL who had been in complete hematological remission. They hypothesized that leukemic cells had infiltrated the retina and choroid, eluding the chemotherapeutic agents and speculated that the blood-brain barrier made it difficult for chemotherapeutic agents to gain ocular entry, allowing chemoresistant clones to survive in the eye.

Our report augments this literature by describing another case of apparent T-ALL relapse localized only to the ocular structures, a case made particularly unusual by the bilateral nature of the patient's presentation. Leukemia should be included in the differential diagnosis as a possible cause of exudative retinal detachment, even among leukemia patients in hematological remission. Our case underscores the fact that the ophthalmologist may be the first provider to detect the relapse of a previously treated leukemia, and that ophthalmic evaluation is critical for detecting malignant ocular infiltrates or ophthalmic complications of systemic chemotherapy.

\section{Statement of Ethics}

The authors have no ethical conflicts to disclose.

\section{Disclosure Statement}

The authors report no conflicts of interests.

\section{References}

1 Sugimoto K-J, Shimada A, Wakabayashi M, Sekiguchi Y, Izumi H, Ota Y, et al: T-cell lymphoblastic leukemia/lymphoma with $\mathrm{t}(7 ; 14)(\mathrm{p} 15 ; \mathrm{q} 32)$ [TCR $\gamma$-TCL1A translocation]: a case report and a review of the literature. Int J Clin Exp Pathol 2014;7:2615-2623.

-2 Russo V, Scott IU, Querques G, Stella A, Barone A, Delle Noci N: Orbital and ocular manifestations of acute childhood leukemia: clinical and statistical analysis of 180 patients. Eur J Ophthalmol 2008;18:619-623.

3 Reddy SC, Jackson N, Menon BS: Ocular involvement in leukemia - a study of 288 cases. Ophthalmologica 2003;217:441-445.

4 Yoshida K, Hasegawa D, Takusagawa A, Kato I, Ogawa C, Echizen N, et al: Bullous exudative retinal detachment due to infiltration of leukemic cells in a child with acute lymphoblastic leukemia. Int J Hematol 2010;92:535-537.

5 Kim J, Chang W, Sagong M: Bilateral serous retinal detachment as a presenting sign of acute lymphoblastic leukemia. Korean J Ophthalmol KJO 2010;24:245-248.

-6 Wu L, Calderón M, Hernández G, Marbis J, Ramírez V: Bilateral exudative retinal detachment as the first sign of relapsing acute myelogenous leukaemia. Clin Experiment Ophthalmol 2006;34:623-625.

-7 Chinta S, Rani PK, Manusani U: Bilateral exudative retinal detachment as a presenting sign of acute lymphoblastic leukemia. Middle East Afr J Ophthalmol 2012;19:410-412.

-8 Azık FM, Akıncı A, Saylı TR, Culha VK, Teberik K, Teke MY, et al: Unilateral exudative retinal detachment as the sole presentation of relapsing acute lymphoblastic leukemia. Turk J Haematol 2012;29:181-184.

-9 Primack JD, Smith ME, Tychsen L: Retinal detachment in a child as the first sign of leukemic relapse: histopathology, MRI findings, treatment, and tumor-free follow up. J Pediatr Ophthalmol Strabismus 1995;32:253-256.

10 Schmiegelow K, Scherfig E, Prause JU, Jensen OA: Isolated leukemic choroidal relapse in a child with acute lymphoblastic leukemia one year off therapy, diagnosed through transvitreal retino-choroidal biopsy. Acta Ophthalmol (Copenh) 1988;66:33-37. 
Case Reports in

Ophthalmology

\begin{tabular}{l|l}
\hline Case Rep Ophthalmol 2015;6:321-327 \\
\hline DOI: $10.1159 / 000439375$ & $\begin{array}{l}\text { (c) } 2015 \text { The Author(s). Published by S. Karger AG, Basel } \\
\text { www.karger.com/cop }\end{array}$ \\
\hline
\end{tabular}

Johnson et al.: Retinal Infiltrate with Exudative Retinal Detachment due to T-ALL Relapse

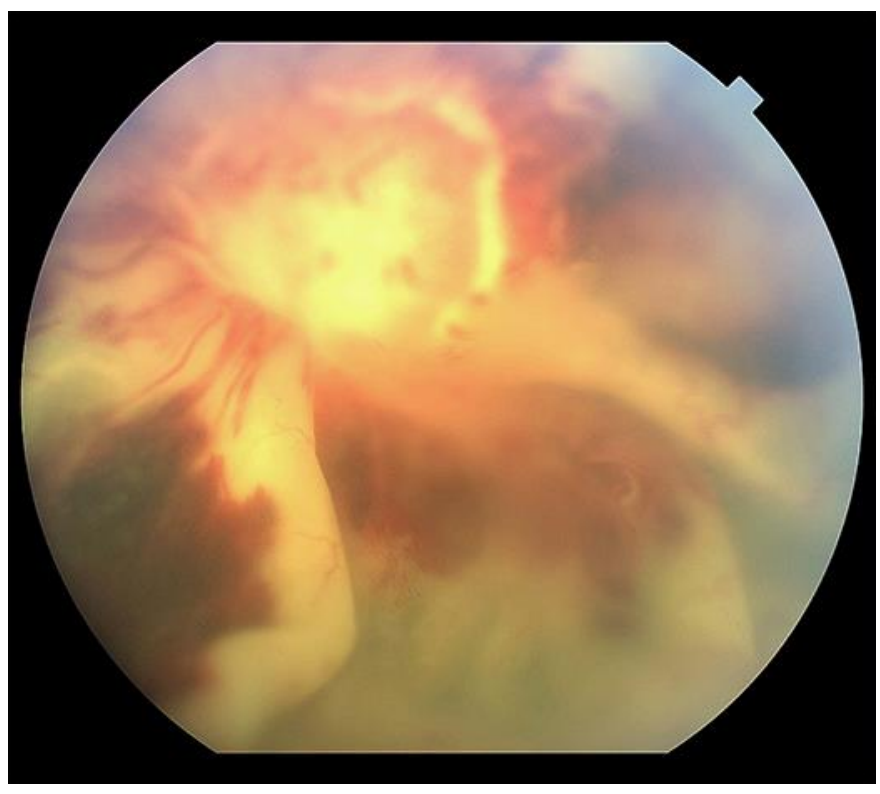

Fig. 1. Diffuse retinal hemorrhaging and whitening of the left eye with areas of bullous elevation and retina folds.

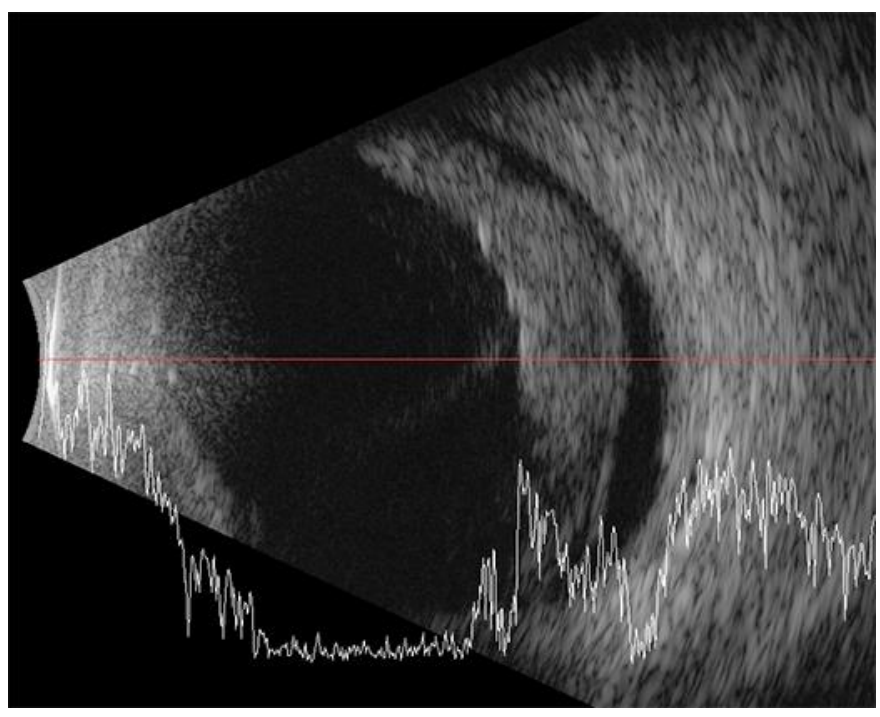

Fig. 2. B-scan ultrasonography showing retinal detachment with significant thickening at the posterior poles. 
Johnson et al.: Retinal Infiltrate with Exudative Retinal Detachment due to T-ALL Relapse

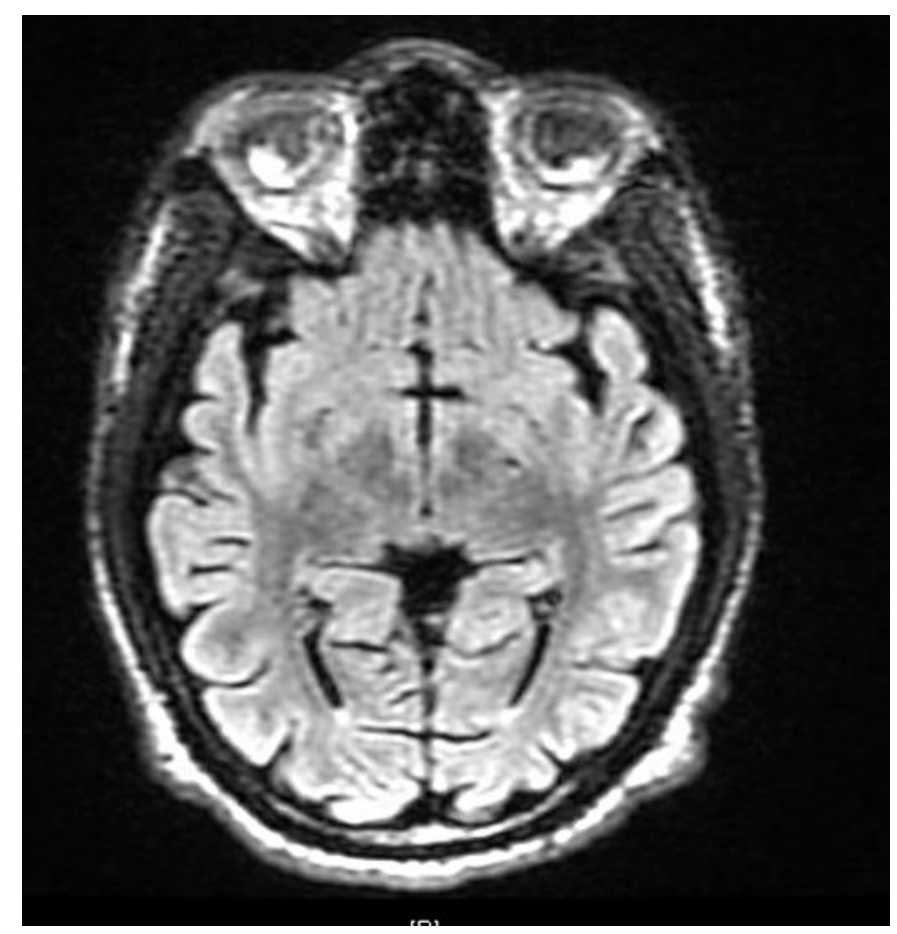

Fig. 3. Axial magnetic resonance imaging showing intraocular hyperintensities of the posterior pole consistent with retinal infiltrate and subretinal exudative fluid. 
Johnson et al.: Retinal Infiltrate with Exudative Retinal Detachment due to T-ALL Relapse

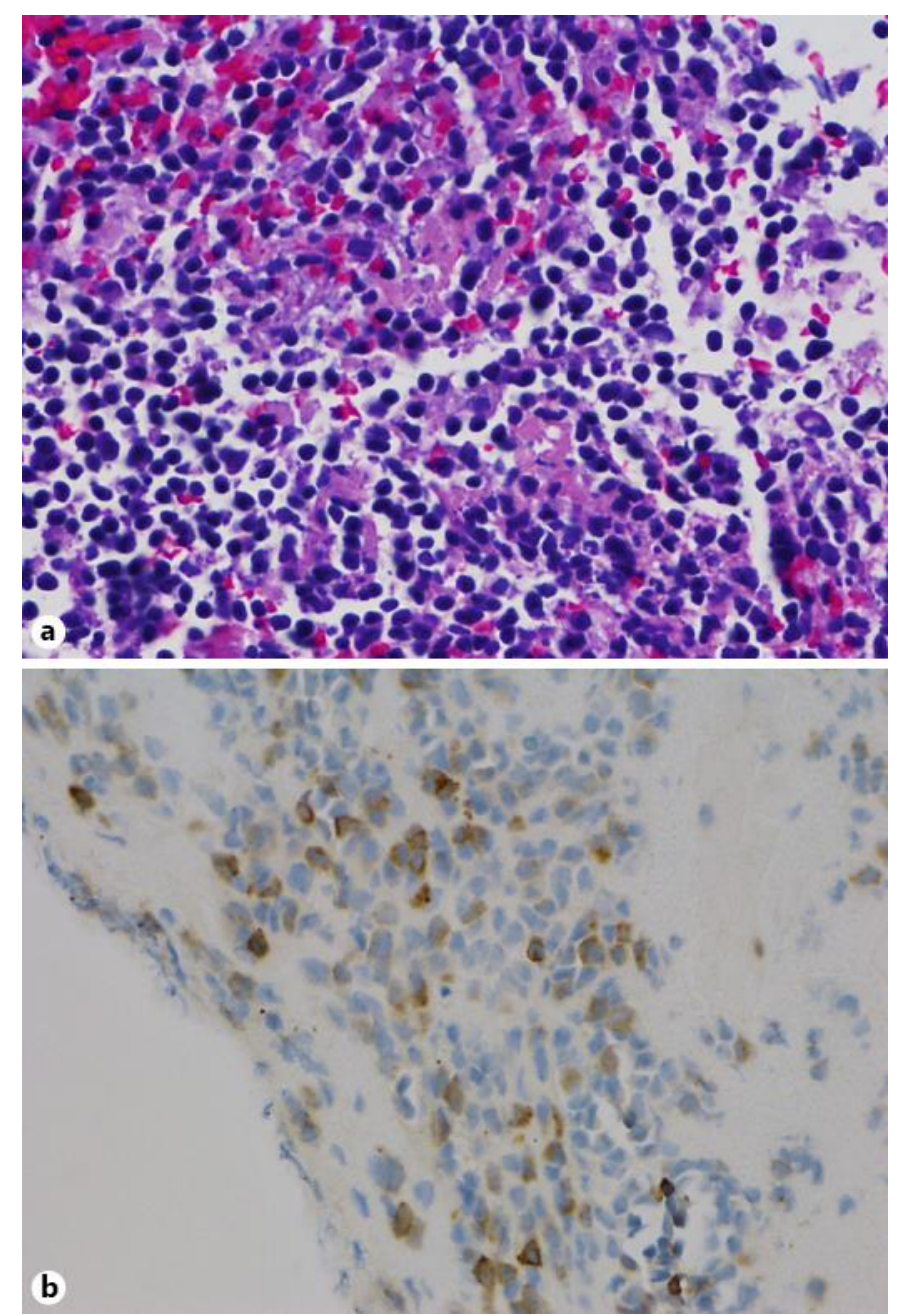

Fig. 4. Retinal biopsy specimen $(\times 400)$ stained with hematoxylin and eosin (a) and CD3 (b). 